Collectanea Mathematica (electronic version): http://www.collectanea.ub.edu

Collect. Math. 61, 1 (2010), 57-63

(C) 2010 Universitat de Barcelona

\title{
Volterra composition operators between weighted Bergman spaces and weighted Bloch type spaces
}

\author{
ELKE WOLF \\ Mathematical Institute, University of Paderborn, D-33095 Paderborn, Germany \\ E-mail: lichte@math.uni-paderborn.de
}

Received January 28, 2009. Revised April 30, 2009

\begin{abstract}
We characterize boundedness and compactness of Volterra composition operators acting between weighted Bergman spaces $A_{v, p}$ and weighted Bloch type spaces $B_{w}$.
\end{abstract}

\section{Introduction}

Let $H(D)$ be the set of all analytic functions on the open unit disk $D$ of the complex plane.

Moreover, let $v$ and $w$ be strictly positive bounded continuous functions (weights) on $D$. Then the weighted Bergman space $A_{v, p}$ is defined as follows

$$
A_{v, p}:=\left\{f \in H(D) ;\|f\|_{v, p}:=\left(\int_{D}|f(z)|^{p} v(z) d A(z)\right)^{1 / p}<\infty\right\}, 1 \leq p<\infty,
$$

where $d A(z)$ is the area measure on $D$ normalized so that area of $D$ is 1 . Moreover we consider the weighted Bloch type spaces $B_{w}$ of functions $f \in H(D)$ satisfying $\|f\|_{B_{w}}:=\sup _{z \in D} w(z)\left|f^{\prime}(z)\right|<\infty$. Provided we identify functions that differ by a constant, $\|\cdot\|_{B_{w}}$ becomes a norm and $B_{w}$ a Banach space.

An analytic self-map $\phi$ of $D$ induces the composition operator $C_{\phi}$ defined by $C_{\phi} f=f \circ \phi$. For an analytic map $g: D \rightarrow \mathbb{C}$ and a map $f \in H(D)$ the Volterra type operator or the Riemann-Stieltjes operator $J_{g}$ is defined as

$$
J_{g} f(z):=\int_{0}^{z} f(\xi) g^{\prime}(\xi) d \xi, z \in D
$$

Keywords: weighted Bergman space, Volterra composition operator, weighted Bloch type space. MSC2000: 47B33, 47B38. 
(see $[15,17])$. In this paper we consider the Volterra composition operator which is defined as follows

$$
\left(J_{g, \phi} f\right)(z):=\int_{0}^{z}(f \circ \phi)(\xi)(g \circ \phi)^{\prime}(\xi) d \xi .
$$

Recently composition operators acting on various spaces of analytic functions have been of much interest, see e.g. [5, 4, 6, 8, 7, 13]. Also operators of type $J_{g}$ have been studied by many authors, see e.g. [1, 2, 3, 15, 17]. Boundedness and compactness of the Volterra composition operator acting between Bergman spaces weighted with the standard weights and Bloch type spaces have been characterized by $\mathrm{Li}$ in [12]. In this article we want to generalize his results for more general weighted Bergman spaces and weighted Bloch type spaces as described above.

\section{Preliminaries}

In the sequel we consider the following weights. Let $\nu$ be a holomorphic function on $D$, non-vanishing, strictly positive on $\left[0,1\left[\right.\right.$ and satisfying $\lim _{r \rightarrow 1} \nu(r)=0$. Then we define the weight $v$ as follows $v(z):=\nu\left(|z|^{2}\right)$ for every $z \in D$.

Next, we give some illustrating examples of weights of this type:

(i) Consider $\nu(z)=(1-z)^{\alpha}, \alpha \geq 1$. Then the corresponding weight is the so-called standard weight $v(z)=\left(1-|z|^{2}\right)^{\alpha}$.

(ii) Select $\nu(z)=e^{-1 /(1-z)^{\alpha}}, \alpha \geq 1$. Then we obtain the weight $v(z)=$ $e^{-1 /\left(1-|z|^{2}\right)^{\alpha}}$.

(iii) Choose $\nu(z)=\sin (1-z)$ and the corresponding weight is given by $v(z)=\sin \left(1-|z|^{2}\right)$.

(iv) Let $\nu(z)=(1-\log (1-z))^{\beta}$ for some $\beta<0$. Then we get $v(z)=(1-\log (1-$ $\left.\left.|z|^{2}\right)\right)^{\beta}$.

For a fixed point $a \in D$ we introduce a function $v_{a}(z):=\nu(\bar{a} z)$ for every $z \in D$. Since $\nu$ is holomorphic on $D$, the function $v_{a}$ is also holomorphic on $D$.

Furthermore, we need some geometric data of the open unit disk. Fix $\alpha \in D$ and consider the authomorphism $\varphi_{\alpha}(z):=\frac{\alpha-z}{1-\bar{\alpha} z}, z \in D$, which interchanges 0 and $\alpha$. Moreover we use the fact that

$$
\varphi_{\alpha}^{\prime}(z)=\frac{|\alpha|^{2}-1}{(1-\bar{\alpha} z)^{2}}, z \in D
$$

Let us finish the preliminaries with stating a very useful lemma, which can be easily derived from [9, Proposition 3.11].

\section{Lemma 1}

Let $v$ and $w$ be weights. Then the operator $J_{g, \phi}: A_{v, p} \rightarrow B_{w}$ is compact if and only if it is bounded and for every bounded sequence $\left(f_{n}\right)_{n}$ in $A_{v, p}$ which converges to zero uniformly on the compact subsets of $D, J_{g, \phi} f_{n}$ tends to zero in $B_{w}$ if $n \rightarrow \infty$. 


\section{Results}

We first need the following auxiliary result. The following lemma is well-known for standard weights (see $[10,11]$ ) and was given in its present form in [16], but for a better understanding we give the full proof here.

\section{Lemma 2}

Let $v$ be a weight as defined in the previous section (i.e. $v(z):=\nu\left(|z|^{2}\right)$ for every $z \in D)$ such that

$$
\sup _{a \in D} \sup _{z \in D} \frac{v(z)\left|v_{a}\left(\varphi_{a}(z)\right)\right|}{v\left(\varphi_{a}(z)\right)} \leq C<\infty \text {. }
$$

Then

$$
|f(z)| \leq \frac{C^{1 / p}}{v(0)^{1 / p}\left(1-|z|^{2}\right)^{2 / p} v(z)^{1 / p}}\|f\|_{v, p}
$$

for all $z \in D, f \in A_{v, p}$.

Proof. Let $\alpha \in D$ be an arbitrary point. Consider the map

$$
T_{\alpha}: A_{v, p} \rightarrow A_{v, p}, T_{\alpha}(f(z))=f\left(\varphi_{\alpha}(z)\right) \varphi_{\alpha}^{\prime}(z)^{2 / p} v_{\alpha}\left(\varphi_{\alpha}(z)\right)^{1 / p} .
$$

Then a change of variables yields

$$
\begin{aligned}
\left\|T_{\alpha} f\right\|_{v, p}^{p} & =\int_{D} v(z)\left|f\left(\varphi_{\alpha}(z)\right)\right|^{p}\left|\varphi_{\alpha}^{\prime}(z)\right|^{2}\left|v_{\alpha}\left(\varphi_{\alpha}(z)\right)\right| d A(z) \\
& =\int_{D} \frac{v(z)\left|v_{\alpha}\left(\varphi_{\alpha}(z)\right)\right|}{v\left(\varphi_{\alpha}(z)\right)}\left|f\left(\varphi_{\alpha}(z)\right)\right|^{p}\left|\varphi_{\alpha}^{\prime}(z)\right|^{2} v\left(\varphi_{\alpha}(z)\right) d A(z) \\
& \leq \sup _{z \in D} \frac{v(z)\left|v_{\alpha}\left(\varphi_{\alpha}(z)\right)\right|}{v\left(\varphi_{\alpha}(z)\right)} \int_{D}\left|f\left(\varphi_{\alpha}(z)\right)\right|^{p}\left|\varphi_{\alpha}^{\prime}(z)\right|^{2} v\left(\varphi_{\alpha}(z)\right) d A(z) \\
& \leq C \int_{D} v(t)|f(t)|^{p} d A(t)=C\|f\|_{v, p}^{p} .
\end{aligned}
$$

Now put $g(z):=T_{\alpha}(f(z))$ for every $z \in D$. By the mean-value property we obtain

$$
v(0)|g(0)|^{p} \leq \int_{D} v(z)|g(z)|^{p} d A(z)=\|g\|_{v, p}^{p} \leq C\|f\|_{v, p}^{p} .
$$

Hence

$$
v(0)|g(0)|^{p}=v(0)|f(\alpha)|^{p}\left(1-|\alpha|^{2}\right)^{2} v(\alpha) \leq C\|f\|_{v, p}^{p}
$$

Thus

$$
|f(\alpha)| \leq C^{1 / p} \frac{\|f\|_{v, p}}{v(0)^{1 / p}\left(1-|\alpha|^{2}\right)^{2 / p} v(\alpha)^{1 / p}} .
$$

Since $\alpha$ was arbitrary, the claim follows.

Calculations show that the examples (i)-(iv) which were listed up above satisfy the assumptions of the previous lemma. 


\section{Theorem 3}

Let $w$ be a weight and $v$ be a weight as in Lemma 2 with

$$
M:=\sup _{a \in D} \sup _{z \in D} \frac{v(z)}{|\nu(\bar{a} z)|}<\infty .
$$

Then the operator $J_{g, \phi}: A_{v, p} \rightarrow B_{w}$ is bounded if and only if

$$
\sup _{z \in D} \frac{w(z)\left|\phi^{\prime}(z)\right| \mid g^{\prime}(\phi(z) \mid}{\left(1-|\phi(z)|^{2}\right)^{2 / p} v(\phi(z))^{1 / p}}<\infty .
$$

Proof. We start with assuming that the operator $J_{g, \phi}$ is bounded. Fix a point $a \in D$ and set

$$
f_{a}(z):=\frac{\varphi_{a}^{\prime}(z)^{2 / p}}{\nu(\bar{a} z)^{1 / p}} \text { for every } z \in D .
$$

Then

$$
\begin{aligned}
\|f\|_{v, p}^{p} & =\int_{D} \frac{\left|\varphi_{a}^{\prime}(z)\right|^{2}}{|\nu(\bar{a} z)|} v(z) d A(z) \\
& \leq \sup _{z \in D} \frac{v(z)}{|\nu(\bar{a} z)|} \int_{D}\left|\varphi_{a}^{\prime}(z)\right|^{2} d A(z) \\
& \leq \sup _{z \in D} \frac{v(z)}{|\nu(\bar{a} z)|} \leq M,
\end{aligned}
$$

and the constant $M$ is independent of the choice of the point $a$. Hence we can find a constant $C^{*}>0$ such that

$$
\begin{aligned}
\frac{w(a)\left|\phi^{\prime}(a)\right|\left|g^{\prime}(\phi(a))\right|}{\left(1-|\phi(a)|^{2}\right)^{2 / p} v(\phi(a))^{1 / p}} & =\left|f_{\phi(a)}(\phi(a))\right| w(a)\left|g^{\prime}(\phi(a))\right|\left|\phi^{\prime}(a)\right| \\
& =\left|\left(J_{g, \phi} f_{\phi(a)}\right)^{\prime}(a)\right| w(a) \leq C^{*}\left\|J_{g, \phi}\right\|\left\|f_{\phi(a)}\right\|_{v, p} .
\end{aligned}
$$

Conversely, we suppose that

$$
\sup _{z \in D} \frac{w(z)\left|\phi^{\prime}(z)\right| \mid g^{\prime}(\phi(z) \mid}{\left(1-|\phi(z)|^{2}\right)^{2 / p} v(\phi(z))^{1 / p}}<\infty .
$$

An application of Lemma 2 yields for $f \in A_{v, p}$

$$
\begin{aligned}
\sup _{z \in D}\left|\left(J_{g, \phi} f\right)^{\prime}(z)\right| w(z) & =\sup _{z \in D}|f(\phi(z))|\left|g^{\prime}(\phi(z))\right|\left|\phi^{\prime}(z)\right| w(z) \\
& \leq \sup _{z \in D} \frac{C^{1 / p}\|f\|_{v, p} w(z)\left|g^{\prime}(\phi(z))\right|\left|\phi^{\prime}(z)\right|}{v(0)^{1 / p}\left(1-\left.\phi(z)\right|^{2}\right)^{2 / p} v(\phi(z))^{1 / p}}
\end{aligned}
$$

Hence the claim follows. 


\section{Theorem 4}

Let $w$ be a weight and $v$ be a weight as in Theorem 3. Then the operator $J_{g, \phi}$ : $A_{v, p} \rightarrow B_{w}$ is compact if and only if

$$
\sup _{z \in D} w(z)\left|g^{\prime}(\phi(z))\right|\left|\phi^{\prime}(z)\right|<\infty
$$

and

$$
\lim _{|\phi(z)| \rightarrow 1} \frac{w(z)\left|g^{\prime}(\phi(z))\right|\left|\phi^{\prime}(z)\right|}{\left(1-|\phi(z)|^{2}\right)^{2 / p} v(\phi(z))^{1 / p}}=0 .
$$

Proof. Assume that the operator $J_{g, \phi}: A_{v, p} \rightarrow B_{w}$ is compact. Then obviously $J_{g, \phi}$ is bounded. Taking $f=1$, we get (0.1). To show $(0.2)$ let $\left(z_{n}\right)_{n}$ be a sequence with $\left|\phi\left(z_{n}\right)\right| \rightarrow 1$ and put

$$
f_{k}(z):=\frac{\varphi_{\phi\left(z_{k}\right)}^{\prime}(z)^{2 / p}}{\nu\left(\overline{\phi\left(z_{k}\right)} z\right)^{1 / p}} \text { for every } z \in D \text { and every } k \in \mathbb{N} .
$$

Analogously to the proof of Theorem 3 we can show that $\left(f_{n}\right)_{n}$ is a bounded sequence which tends to zero uniformly on the compact subsets of $D$. Since $J_{g, \phi}$ is compact, by Lemma 1

$$
\left\|J_{g, \phi} f_{n}\right\|_{B_{w}} \rightarrow 0 \text { if } n \rightarrow \infty
$$

Thus,

$$
\left\|J_{g, \phi} f_{n}\right\|_{B_{w}} \geq \frac{w\left(z_{n}\right)\left|\phi^{\prime}\left(z_{n}\right)\right| \mid g^{\prime}\left(\phi\left(z_{n}\right) \mid\right.}{\left(1-\left|\phi\left(z_{n}\right)\right|^{2}\right)^{2 / p} v\left(\phi\left(z_{n}\right)\right)^{1 / p}},
$$

and condition (0.2) follows.

Conversely, suppose that (0.1) and (0.2) are satisfied. Let $\left(f_{n}\right)_{n}$ be a bounded sequence in $A_{v, p}$ such that $\left\|f_{n}\right\|_{v, p} \leq M_{1}<\infty$ for every $n \in \mathbb{N}$ and such that $\left(f_{n}\right)_{n}$ converges uniformly to zero on the compact subsets of $D$ if $n \rightarrow \infty$. For a fixed $\varepsilon>0$ we can find $0<r_{0}<1$ such that if $|\phi(z)|>r_{0}$, then

$$
\frac{w(z)\left|g^{\prime}(\phi(z))\right|\left|\phi^{\prime}(z)\right|}{\left(1-|\phi(z)|^{2}\right)^{2 / p} v(\phi(z))^{1 / p}}<\frac{\varepsilon v(0)^{1 / p}}{2 C^{1 / p} M_{1}}
$$

Moreover, we can find $M_{2}>0$ such that

$$
\sup _{|\phi(z)| \leq r_{0}} w(z)\left|g^{\prime}(\phi(z))\right|\left|\phi^{\prime}(z)\right| \leq M_{2} .
$$

There is $n_{0} \in \mathbb{N}$ such that

$$
\sup _{|\phi(z)| \leq r_{0}}\left|f_{n}(\phi(z))\right| \leq \frac{\varepsilon}{2 M_{2}} \text { for every } n \geq n_{0} .
$$

Furthermore, from (0.2) we can easily derive that

$$
\sup _{z \in D} \frac{w(z)\left|\phi^{\prime}(z)\right| \mid g^{\prime}(\phi(z) \mid}{\left(1-|\phi(z)|^{2}\right)^{2 / p} v(\phi(z))^{1 / p}}<\infty .
$$


Thus, the operator $J_{g, \phi}$ must be bounded. We obtain applying Lemma 2

$$
\begin{aligned}
\sup _{z \in D}\left|\left(J_{g, \phi} f_{n}\right)^{\prime}(z)\right| w(z)= & \sup _{z \in D} w(z)\left|f_{n}(\phi(z))\right|\left|g^{\prime}(\phi(z))\right|\left|\phi^{\prime}(z)\right| \\
\leq & \sup _{|\phi(z)| \leq r_{0}} w(z)\left|f_{n}(\phi(z))\right|\left|g^{\prime}(\phi(z))\right|\left|\phi^{\prime}(z)\right| \\
& +\sup _{|\phi(z)|>r_{0}} w(z)\left|f_{n}(\phi(z))\right|\left|g^{\prime}(\phi(z))\right|\left|\phi^{\prime}(z)\right| \\
\leq & \sup _{|\phi(z)| \leq r_{0}}\left|f_{n}(\phi(z))\right| \sup _{|\phi(z)| \leq r_{0}} w(z)\left|g^{\prime}(\phi(z))\right|\left|\phi^{\prime}(z)\right| \\
& +\sup _{|\phi(z)|>r_{0}} \frac{C^{1 / p}\left\|f_{n}\right\|_{v, p} w(z)\left|g^{\prime}(\phi(z))\right|\left|\phi^{\prime}(z)\right|}{v\left(1-|\phi(z)|^{2}\right)^{2 / p} v(\phi(z))^{1 / p}} \\
\leq & \varepsilon,
\end{aligned}
$$

and the claim follows.

\section{References}

1. A. Aleman and J.A. Cima, An integral operator on $H^{p}$ and Hardy's inequality, J. Anal. Math. 85 (2001), 157-176.

2. A. Aleman and A.G. Siskakis, An integral operator on $H^{p}$, Complex Variables Theory Appl. 28 (1995), 149-158.

3. A. Aleman and A.G. Siskakis, Integration operators on Bergman spaces, Indiana Univ. Math. J. 46 (1997), 337-356.

4. J. Bonet, P. Domański, and M. Lindström, Essential norm and weak compactness of composition operators on weighted Banach spaces of analytic functions, Canad. Math. Bull. 42 (1999), 139-148.

5. J. Bonet, P. Domański, M. Lindström, and J. Taskinen, Composition operators between weighted Banach spaces of analytic functions, J. Austral. Math. Soc. Ser. A 64 (1998), 101-118.

6. J. Bonet, M. Friz, and E. Jordá, Composition operators between weighted inductive limits of spaces of holomorphic functions, Publ. Math. Debrecen 67 (2005), 333-348.

7. J. Bonet, M. Lindström, and E. Wolf, Differences of composition operators between weighted Banach spaces of holomorphic functions, J. Austral. Math. Soc. 84 (2008), 9-20.

8. M.D. Contreras and A.G. Hernández-Díaz, Weighted composition operators in weighted Banach spaces of analytic functions, J. Austral. Math. Soc. Ser. A 69 (2000), 41-60.

9. C.C. Cowen and B.D. MacCluer, Composition Operators on Spaces of Analytic Functions, Studies in Advanced Mathematics, CRC Press, Boca Raton, FL, 1995.

10. P. Duren and A. Schuster, Bergman Spaces, Mathematical Surveys and Monographs 100, American Mathematical Society, Providence, RI, 2004.

11. H. Hedenmalm, B. Korenblum, and K. Zhu, Theory of Bergman Spaces, Graduate Texts in Mathematics 199, Springer-Verlag, New York, 2000.

12. S. Li, Volterra composition operators between weighted Bergman spaces and Bloch type spaces, J. Korean Math. Soc. 45 (2008), 229-248.

13. M. Lindström and E. Wolf, Essential norm of the difference of weighted composition operators, Monatsh. Math. 153 (2008), 133-143.

14. J.H. Shapiro, Composition Operators and Classical Function Theory, Tracts in Mathematics, Springer-Verlag, New York, 1993. 
15. A.G. Sisakis and R. Zhao, A Volterra type operator on spaces of analytic functions, Functions spaces (Edwardville IL, 1998), 299-311, Contemp. Math. 232, Amer. Math. Soc. Providence, RI, 1999.

16. E. Wolf, Weighted composition operators between weighted Bergman spaces, Rev. R. Acad. Cien. Ser. A Mat. 103 (2009), 11-15.

17. J. Xiao, Riemann-Stieltjes operators on weighted Bloch and Bergman spaces of the unit ball, $J$. London, Math. Soc. (2) 70 (2004), 199-214. 